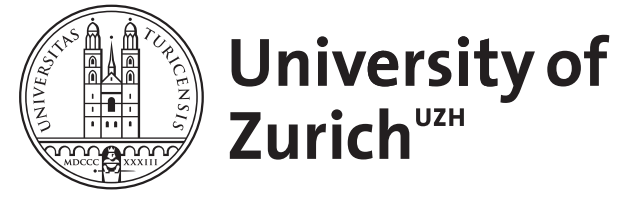

\title{
Einleitung: Wissensgeschichte ökonomischer Praktiken
}

\author{
Dommann, Monika ; Speich Chassé, Daniel ; Suter, Mischa
}

DOI: https://doi.org/10.1002/bewi.201401683

Posted at the Zurich Open Repository and Archive, University of Zurich ZORA URL: https://doi.org/10.5167/uzh-96719

Journal Article

Published Version

Originally published at:

Dommann, Monika; Speich Chassé, Daniel; Suter, Mischa (2014). Einleitung: Wissensgeschichte ökonomischer Praktiken. Berichte zur Wissenschaftsgeschichte, 37(2):107-111.

DOI: https://doi.org/10.1002/bewi.201401683 


\section{Einleitung:}

\section{Wissensgeschichte ökonomischer Praktiken}

Wie in der Medizin und der Rechtswissenschaft bis heute üblich, mussten künftige Ökonomen und Ökonominnen bis vor kurzem im Rahmen ihres Studiums auch Geschichtsvorlesungen besuchen. Im deutschen Sprachraum hieß dieses Pflichtfach abschreckend „Dogmengeschichte“, in der angelsächsischen Tradition war eleganter von „History of Economic Thought“ die Rede. Die Dogmengeschichte fokussiert die Evolution von Ideen im Dienste ökonomischer Theoriebildung und Ausbildung. Die folgenden Aufsätze schließen zwar an jene dogmengeschichtliche Traditionen an, aber sie verfolgen keine Geschichte von ökonomischen Ideen, sondern eine Wissensgeschichte ökonomischer Praktiken. ${ }^{1}$ Damit ist eine Perspektivierung intendiert, welche die Genese und Transformation von Wissen als Praxen in Gemengelagen untersucht, das heißt in jenen Zonen, wo Wirtschaft, Politik, Recht, Film, Literatur und Theater aufeinander wirken, sich bedingen, sich stören und zuweilen auch lose nebeneinander koexistieren. Dabei kommen zwei Analyseebenen ins Spiel: erstens die Betrachtung von Kapitalismus als spezifische Form des Tausches und zweitens die Rolle von Kultur- und Medientechniken (wie Klassifikationen, Statistiken, Erzähltechniken) als Beschreibungsmodus, Analyseraster und Interventionstechnik ökonomischer Praktiken. ${ }^{2}$

Wirtschaftliche Studien, die begrifflich beim Kapitalismus ansetzen, erleben gegenwärtig eine Renaissance, wobei dem Begriff die Konnotation der Turbulenz, Störungsanfälligkeit, Krisenhaftigkeit und Instabilität anhaftet. ${ }^{3}$ Dass dem Konzept ,Kapitalismus' gleichzeitig die Funktion von Analyse und Kritik zukommt, ist wissenshistorisch interessant. ${ }^{4}$ Diese Analyse und Kritik bezogen sich auf einen Gegenstand, dessen Konturen selbst stets strittig waren. Denn zum einen verkomplizierte unpersönlicher, geldvermittelter Austausch, wie er für den Kapitalismus charakteristisch ist, die politische Frage nach der Macht. Wessen Kopf sollte abgeschlagen werden, wenn kein König, sondern ein prozessierendes Verhältnis die soziale Ordnung aufrechterhielt? ${ }^{5}$ Zum anderen war die Ökonomie im 18. Jahrhundert, dem Moment, als sie ihre eigenen Wissensbestände ausformte, bereits kapitalistisch: für die Politische Ökonomie beinhaltete eine Beschreibung der Wirtschaft immer schon eine Analytik des Kapitalismus. ${ }^{6}$ Die Frage nach der Gestalt des Ökonomischen hat den Kapitalismus seither begleitet, auch und gerade wenn die Wirtschaftswissenschaften sich ihrer zu entledigen suchten, etwa wenn Robbins die Ökonomie als „human behaviour in disposing of scarce means “ definitorisch universalisierte. ${ }^{7}$ Die klassische Politische Ökonomie ließ ein Figurenpersonal des Kapitalisten, des Arbeiters und des Grundbesitzers auftreten. Aus diesem Ensemble war die nunmehr weiblich deklarierte Hausarbeit konstitutiv ausgeblendet. ${ }^{8}$ Mit dem Aufstieg der Grenznutzenschule rückten diese Figuren in den Hintergrund, und die Ökonomie formierte 
sich neu als Wissenschaft des formalisierten, subjektiven Tausches: aus „political economy“ wurden „economics“. ${ }^{9}$ Die Bewegung des Austauschs stand auch hinter der Über-Metapher der deutschsprachigen Wirtschaftswissenschaft im Liberalismus, dem „Verkehr“. ${ }^{10}$ Tausch stand aber stets auch in einem Spannungsfeld zu weiteren zeitgenössischen Konzepten, wie der Produktion. Heute, in der neoliberalen Gegenwart, haben Tausch und Zirkulation erneut verstärkte Virulenz in zeitgenössischen Semantiken erlangt. Doch zeigt die Geschichte ökonomischer Diskurse und Leitkonzepte auch, welche fatalen Folgen die einseitige Verabsolutierung von Tausch hatte. Die politische Aufladung vom ,Mittelsmann' als Ausbeuter und der Zirkulationssphäre als Domäne der Ausbeutung fand in antisemitischen Feindbildkonstruktionen ihren folgenschwersten Ausdruck.

Kurz gefasst: Eine Wissensgeschichte des kapitalistischen Tauschs widmet sich den Szenen und Figurationen, in die das opake Objekt Wirtschaft eingefasst wurde. Dabei geht Wissensgeschichte nicht in einer Geschichte der Repräsentation ökonomischer Praktiken auf, sondern stellt den Status des Untersuchungsobjekts selbst zur Disposition. ${ }^{11}$ Wenn ökonomischen Wirkkräften im Kapitalismus eine eigene Stellung zukommt, dann lässt sich nach den Wissensformen fragen, aus denen sich dieser Status des Ökonomischen aufbaute. ${ }^{12}$ Statt dem Untersuchungsgegenstand Kapitalismus wahlweise mit einer wirtschaftsgeschichtlichen oder kulturhistorischen Deutung zu begegnen, lädt die Wissensgeschichte dazu ein zu fragen, was denn das Ökonomische oder das Kulturelle einer solchen Deutung wäre, was sie erhellen oder verdunkeln würde. Insofern ist die hier vorgeschlagene Wissensgeschichte ein reflexives Unterfangen, das zugleich über sich selbst hinausweist. Karl Marx’ Kritik der Politischen Ökonomie hat ein solches Projekt gebildet, an das eine Wissensgeschichte ökonomischer Praktiken anknüpfen und dabei Geschichte als eine Wissenschaft der Kritik betreiben kann. ${ }^{13}$ Kritik formuliert, so verstanden, nicht Einspruch gegen diese oder jene Unzulänglichkeiten eines Theoriegebäudes, sondern sie zielt auf das Ganze, das heißt auf das Zusammenspiel der fundierenden Annahmen und der Ausblendungen eines Wissensbestands. ${ }^{14}$ Kritik ,ent-selbstverständlicht' ${ }^{\dagger}$, indem sie die innere Logik eines Normalbetriebs nach außen kehrt und Konstitutionsbedingungen aufspürt, die die alltäglichsten Praktiken durchwirken. Wissenschaftsgeschichtlich hat die historische Epistemologie einen ähnlichen detaillierten Blick auf Haltungen und Praktiken erprobt. Doch bleibt Kapitalismus ein Makrokonzept, das nur schwer in eine feinkörnige Analyse von Wissenspraktiken einbezogen werden kann. Und umgekehrt ist gegenüber Studien der historischen Epistemologie eingewandt worden, dass die detaillierte Analyse epistemischer Praktiken auf Kosten der Analyse weiterer Kräfte gehe, die auf das Labor oder das Untersuchungsfeld einwirken. ${ }^{15}$ Die Grenzen des ethnografischen Felds erscheinen dann als Grenzen der Welt. ${ }^{16}$ Eine Wissensgeschichte ökonomischer Praktiken gewinnt, wenn sie diese Einwände berücksichtigt. Dabei kommt ihr letztlich die Anlage ihres Untersuchungsobjekts, die kapitalistische Ökonomie, entgegen, denn das entsprechende Wissen hielt sich zu keinem Zeitpunkt an die disziplinären Grenzen der Wirtschaftswissenschaften. ${ }^{17}$ Wenn sie als Herrschaftstechnologie betrieben wird, ist die Ökonomie stets auf einem ,Gang durch die Gesellschaft ${ }^{6}{ }^{18}$ Für die historische Rekonstruktion liegt eine Herausforderung darin, einen übergeordneten Analyserahmen wie Kapitalismus anzunehmen, ohne aber durch die Setzung dieses Rahmens die konkrete Untersuchung vorweg zu nehmen. Vom Konkreten her ist die Indeterminanz in einem System nachzuzeichnen. 
Ein Vorschlag zur Lösung dieses Problems ist, Konflikte ins Zentrum der Analyse zu stellen und deren epistemische Dimension freizulegen, indem gezeigt wird, wie historische Akteure über den Status ihres Streitgegenstands selbst stritten. Denn schließlich baut sich ökonomisches Wissen zutiefst konfliktträchtig auf. Kritiken der Politischen Ökonomie haben auf die Rolle der Gewalt in der Formatierung von Praktiken und Wissen hingewiesen, um Privateigentum, Vertrag und letztlich generell: kapitalistischen Tausch einzurichten. ${ }^{19}$

Die Wissensgeschichte von ökonomischen Praktiken muss versuchen, die Mikroanalyse der Herstellung von Erkenntnis mit der Analyse ihrer gesellschaftlichen Orte und Wirkungen zu verbinden. Sie ist deshalb besonders interessiert an Fragen der Materialität und der Repräsentation. Wenn Konflikte von ihren lokalen Schauplätzen her in einem System von Perturbationen, dem Kapitalismus, situiert werden, öffnet sich die Kapitalismusanalyse auch der präzisen Untersuchung von medienund kulturtechnischen Arrangements, in denen ökonomisch handelnde Subjekte verhaftet waren. Hier finden die zwei Analyseebenen zusammen: die spezifische Form des kapitalistischen Tausches hat ihre große gesellschaftliche Wirkmacht durch besondere Repräsentationsformen des Ökonomischen erlangt. Heute ist das Wissen über die Wirtschaft von dieser Wirtschaft selbst nicht zu trennen. Die Rede von einer „knowledge-based economy“ beziehungsweise von einer Wissensgesellschaft bringt diesen engen Zusammenhang zum Ausdruck. Aber er besteht schon lange und prägte auch das produktionsorientierte Paradigma des fordistischen Kapitalismus.

Die Beiträge zu diesem Heft interessieren sich für erfolgreiche und gescheiterte Versuche, Wissen, Güter und Werte herzustellen und zirkulieren zu lassen. Dabei gehen sie nicht von einer fundamentalen Trennung zwischen der Materialität des Wirtschaftslebens und seiner diskursiven Überformung aus. Vielmehr geht es gerade darum, die Verschränkung dieser zwei Dimensionen nachzuzeichnen. Dabei ist freilich eine andere Unterscheidung sorgfältig in Betracht zu ziehen: jene zwischen der ,economy', der Wirtschaft, und den ,economics', dem Wissen darüber. In beiden steckt sowohl Materielles als auch Diskursives: die Wirtschaft, the ,economy', ist nicht nur verdichtete Materialität, sondern in erster Linie ein Wissensobjekt; und die Wirtschaftswissenschaft, ,economics', ist nicht nur geistiges Treiben, sondern konkrete Arbeit und Schauplatz von Kämpfen. Kurzum: Der hier vorgestellte Ansatz einer Wissensgeschichte ökonomischer Praktiken erweist sich als ein Forschungsprogramm, das weiterzuverfolgen sich unserer Meinung nach lohnt und zu dessen Vertiefung wir anregen möchten.

In ihrer programmatischen Einleitung zum Sammelband Bild, Schrift, Zabl aus dem Jahr 2003 sprachen die Herausgeber Sibylle Krämer und Horst Bredekamp von Kultur und meinten damit Kulturtechniken. ${ }^{20}$ Es ging ihnen und all jenen KulturwissenschaftlerInnen, welche diesen Ingenieursbegriff aus dem 19. Jahrhundert zweckentfremdet haben, um den Umgang mit und die Kultivierung von Sachen. Damit sind Techniken, Riten, Fertigkeiten und Praktiken mitgemeint und neben Sprache insbesondere auch fotografische, kinematografische und televisionäre Bilder sowie die Formalisierung der modernen Wissenschaften durch die Mathematik. Eine Wissensgeschichte ökonomischer Praktiken ist aus dreierlei Gründen immer auch als eine Geschichte kulturtechnischer Operationen zu analysieren: ${ }^{21}$ Erstens operiert die Wirtschaftswissenschaft mit Modellen.22 Seit François Quesnays Tableau Economique aus dem 18. Jahrhundert ist Modellbildung auch Bildproduktion. Es 
handelt sich dabei um Akte der Imagination, um epistemische Objekte, zuweilen gar um Techniken wissenschaftlicher Theoriebildung, um materielle Objekte manchmal obendrein, aber genauso auch um Herrschaftstechniken der Klassifikation, wenn hiermit Inklusion und Exklusion betrieben und dabei soziale Tatsachen geschaffen werden. Zweitens operiert ökonomische Wissensproduktion seit gut 200 Jahren mehr oder weniger erfolgreich mit Operationen, die Ian Hacking mit dem Begriff „statistischer Inferenz" umschrieben hat. ${ }^{23}$ Es handelt sich um Techniken des Zugriffs auf kurze, längere oder sehr lange Zeiträume und um Operationen, die danach zielen, individuelle Irregularität mit Praktiken aggregierter Regularität zu kombinieren. Die Evidenzerzeugung solcher Wissensformationen sind immer Zukunftsprojekte. Doch dessen ungeachtet sind sie immer imstande mit Gesellschaften zu interferieren, weil individuelles Handeln vor diesem Hintergrund auch ein Handeln mit dem Wissen um aggregierte Zustände einschließt. Drittens sind ökonomische Praktiken mit Problemen der Sichtbarkeit, der Darstellbarkeit und der Erzählbarkeit behaftet. Eine Wissensgeschichte der Ökonomie handelt deshalb immer auch von einer Poetologie des Wissens der Ökonomie. ${ }^{24}$ Welche Register dabei gezogen werden, ist nicht zufällig und deshalb analysewürdig. Dass mit toten Ökonomen neues Wissen zu gewinnen sei, behauptet jedenfalls ein ökonomischer Bestseller. ${ }^{25}$ Geschichtsvergessen scheint die geisteraffine Ökonomie also noch nicht zu sein.

\section{Die Herausgeberin und die Herausgeber danken Cornelius Borck} für Begeisterung, Kritik und Geduld.

1 Zur Konzeption einer Wissensgeschichte vgl. Daniel Speich Chassé, David Gugerli, Wissensgeschichte. Eine Standortbestimmung, Traverse 1 (2012), 85-100; Philipp Sarasin, Was ist Wissensgeschichte?, Internationales Archiv für Sozialgeschichte der deutschen Literatur 36, 1 (2011), 159-172.

2 Vgl. hierzu: Joseph Vogl, Kalkül und Leidenschaft. Poetik des ökonomischen Menschen, München: Sequenzia 2002; derselbe, Das Gespenst des Kapitals, Zürich: Diaphanes 2010; Urs Stäheli, Spektakuläre Spekulation. Das Populäre in der Ökonomie, Frankfurt am Main: Suhrkamp 2007.

3 Vgl. hierzu: Jürgen Kocka, Kommentar: Kapitalismus im Kontext, in: Gunilla Budde (Hrsg.), Kapitalismus. Historische Annäherungen, Göttingen: Vandenhoeck \& Ruprecht 2011, S. 176-188.

4 Jürgen Kocka, Geschichte des Kapitalismus, München: C. H. Beck 2013, S. 6-9, hier S. 9. Das Charakteristikum Wissenschaft und Gesellschaftskritik explizit zu vereinen, teilt die Kapitalismusanalyse übrigens mit anderen Wissensformationen der Sozial- und Geisteswissenschaften, wie etwa der aktuellen Genderforschung und den Postcolonial Studies.

5 Hier und im Folgenden: Susan Buck-Morss, Envisioning Capital: Political Economy on Display, Critical Inquiry 21 (1995), 434-467, hier S. 437-439.

6 Buck-Morss, Envisioning (wie Anm. 5), S. 437-439. Dazu auch Immanuel Wallerstein, The Modern World System, 4 Bände, Berkeley: University of California Press 1974-2010; Giovanni Arrighi, Adam Smith in Beijing. Die Genealogie des 21. Jahrbunderts, Hamburg: VSA 2008.

7 Vgl. Lionel Robbins, An Essay on the Nature E Siginificance of Economic Science, London: Macmillan 1952 [London 1932], S. 15.

8 Klassisch dazu: Gisela Bock, Barbara Duden, Arbeit aus Liebe - Liebe als Arbeit, in: Gruppe Berliner Dozentinnen (Hrsgg.), Frauen und Wissenschaft. Beiträge zur Berliner Sommeruniversität für Franen, Juli 1976, Berlin (West): Courage 1977, S. 118-199; Mariarosa Dalla Costa, Selma James, Die Macht der Frauen und der Umsturz der Gesellschaft, Berlin (West): Merve 1973.

9 Keith Tribe, Continental Political Economy from the Physiocrats to the Marginal Revolution, in: Theodore M. Porter (Hrsg.), The Cambridge History of Science, Band 7: The Modern Social Sciences, Cambridge: Cambridge University Press 2003, S. 154-170, hier S. 154; aus new historicist-Perspektive zu diesem Wandel Regenia Gagnier, The Insatiability of Human Wants: Economics and Aesthetics in Market Society, Chicago: University of Chicago Press 2000. 
10 Keith Tribe, Strategies of Economic Order: German Economic Discourse, 1750-1950, Cambridge: Cambridge University Press 1995, S. 73.

11 Für eine analoge Diskussion in der Sozialgeschichte über den Status deren Untersuchungsobjekts ,Gesellschaft' vgl. Patrick Joyce, What is the Social in Social History?, Past and Present 206 (2010), $213-248$.

12 Vgl. beispielsweise zur Frage, was den Status eines ökonomischen Ereignisses ausmacht, bei William Sewell, Economic Crises and the Shape of Modern History, Public Culture 24 (2012), 303-327.

13 Karl Marx, Das Kapital. Kritik der politischen Ökonomie, 3 Bände, Berlin (DDR): Dietz 1971 (= MEW, Bde. 23-25); derselbe, Grundrisse der Kritik der politischen Ökonomie, Berlin (DDR): Dietz 1983 (= MEW, Bd. 42).

14 Dezidiert zu Geschichte als Wissenschaft der Kritik: Joan W. Scott, History-writing as Critique, in: Keith Jenkins u.a. (Hrsgg.), Manifestos for History, London usw.: Routledge 2007, S. 19-38, besonders S. 23-26.

$15 \mathrm{Zu}$ dieser Debatte unlängst in der vorliegenden Zeitschrift: Volker Roelcke, Auf der Suche nach der Politik in der Wissensproduktion: Plädoyer für eine historisch-politische Epistemologie, Berichte zur Wissenschaftsgeschichte 33 (2010), 176-192; Katherina Kinzel, Geschichte ohne Kausalität. Abgrenzungsstrategien gegen die Wissenschaftssoziologie in zeitgenössischen Ansätzen historischer Epistemologie, Berichte zur Wissenschaftsgeschichte 35 (2012), 147-162; Dominique Pestre, Regimes of Knowledge Production in Society: Towards a More Political and Social Reading, Minerva 41, 3 (2003), $245-261$.

16 Für eine in mancher Hinsicht parallele Diskussion in den Social Studies of Finance vgl. Annelise Riles, Collateral Expertise: Legal Knowledge in the Global Financial Markets, Current Anthropology 51, 6 (2010), 795-818 und die entsprechenden Gastkommentare zu diesem Aufsatz.

17 Michel Callon, Introduction: The Embeddedness of Economic Markets in Economics, in: derselbe (Hrsg.), The Lawe of the Markets, Oxford: Blackwell 1998, S. 1-57.

18 Timothy Mitchell, Rule of Experts. Egypt, Techno-Politics, Modernity, Berkeley: University of California Press 2002.

19 Mitchell, Rule (wie Anm. 18). Vgl. auch David Harveys Konzept der „Akkumulation durch Enteignung“, das Marx’ Begriff der „so genannten ursprünglichen Akkumulation“ adaptiert: David Harvey, The New Imperialism, Oxford: Oxford University Press 2003; Marx, Kapital Bd. 1, Kapitel 24 (wie Anm. 13): „Die sogenannte ursprüngliche Akkumulation“.

20 Sibylle Krämer, Horst Bredekamp, Kultur, Technik, Kulturtechnik. Wider die Diskursivierung der Kultur, in: dieselben (Hrsgg.), Bild, Schrift, Zabl, Zürich: Wilhelm Fink 2003, S. 11-22.

21 Vgl. hierzu auch: Anna Echterhölter, Dietmar Kammerer, Rebekka Ladewig (Hrsgg.), Ökonomische Praktiken, ilinx - Berliner Beiträge zur Kulturwissenschaft 3 (2013), Berlin: Philo Fine Arts 2013.

22 Mary S. Morgan, Seeking Parts, Looking for Wholes, in: Lorraine Daston, Elizabeth Lunbeck (Hrsgg.), Histories of Scientific Observation, Chicago/London: University of Chicago Press 2011, S. 303-325. Mary S. Morgan, The World and the Model. How Economists Work and Think, Cambridge: Cambridge University Press 2012.

23 Ian Hacking, Logic of Statistical Inference, Cambridge: Cambridge University Press 1976 [Cambridge 1965].

24 Vgl. hierzu: Joseph Vogl (Hrsg.), Poetologien des Wissens um 1800, München: Wilhelm Fink 1999.

25 Todd Buchholz, New Ideas from Dead Economists: An Introduction to Modern Economic Thought, New York: Penguin 1999.

Anschriften der Verfasserin und der Verfasser: Prof. Dr. Monika Dommann, Historisches Seminar, Universität Zürich, Karl-Schmid Straße 4, CH-8006 Zürich, E-Mail: monika.dommann@hist.uzh.ch; Prof. Dr. Daniel Speich Chassé, SNF-Förderprofessor für neuere und neueste Geschichte, Universität Luzern, Historisches Seminar, Frohburgstrasse 3, CH-6002 Luzern, E-Mail: daniel.speich@unilu.ch; Dr. des. Mischa Suter, Departement Geschichte Universität Basel, Hirschgässlein 21, CH-4001 Basel, E-Mail: mischa.suter@unibas.ch 